\title{
ON THE 75TH BIRTHDAY OF PROFESSOR VITALII VLADIMIROVICH ARESTOV
}

\section{Vitalii I. Berdyshev ${ }^{\dagger}$, Nikolai I. Chernykh ${ }^{\dagger \dagger}$, Alexander G. Babenko ${ }^{\dagger \dagger}$, and Roman R. Akopyan ${ }^{\dagger \dagger \dagger \dagger}$}

Krasovskii Institute of Mathematics and Mechanics, Ural Branch of the Russian Academy of Sciences, 16 S. Kovalevskaya str., Ekaterinburg, Russia, 620990;

Ural Federal University, 51 Lenin aven., Ekaterinburg, Russia, 620000

†bvi@imm.uran.ru, ††chernykh@imm.uran.ru, ${ }^{\dagger} \dagger$ babenko@imm.uran.ru,

†t†'rrakopyan@mephi.ru

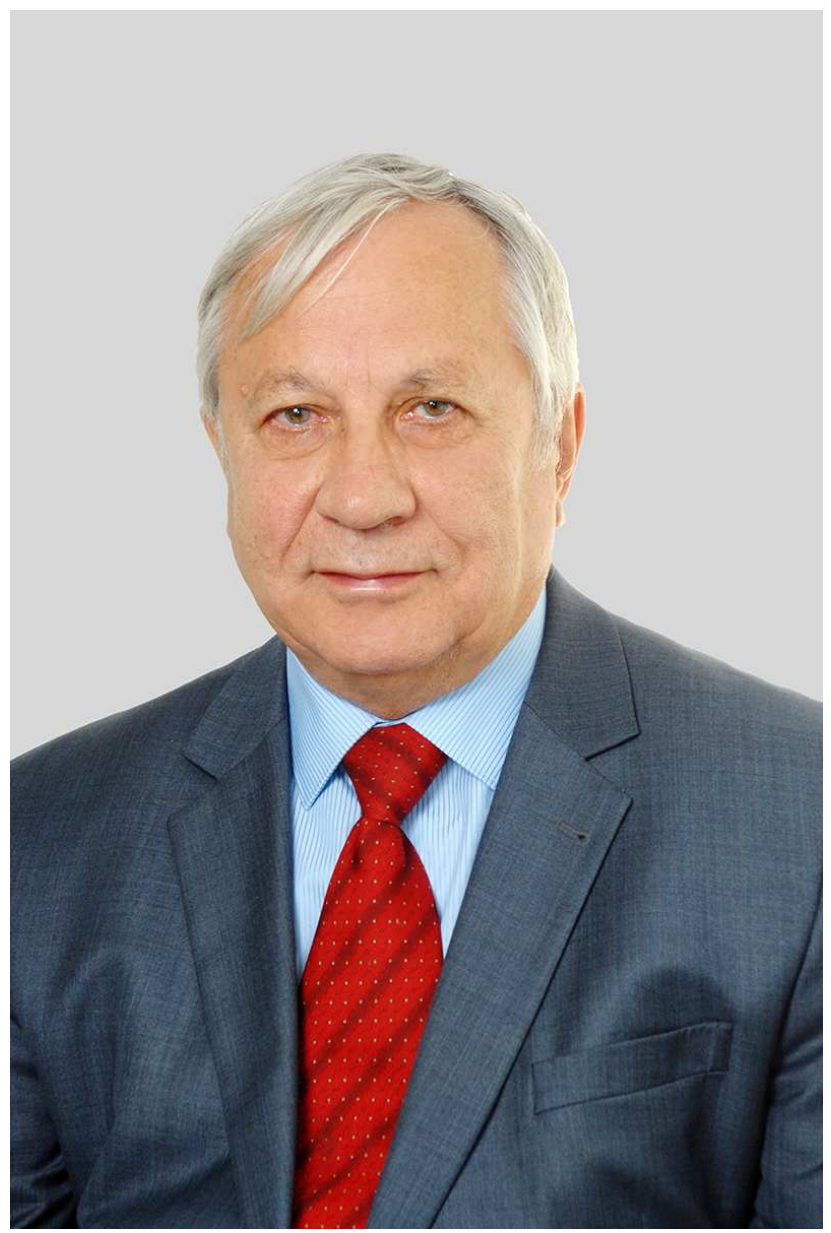

Figure 1. Professor Vitalii Vladimirovich Arestov

July 16, 2018, was the 75th birthday of famous Russian scientist, prominent mathematician, Doctor of Physics and Mathematics, Professor Vitalii Vladimirovich Arestov. 
Arestov was born in the village Bol'shoi Karai (Saratov oblast). He graduated from Saratov State University as specialist in mathematics and completed postgraduate studies in Steklov Mathematical Institute of the USSR Academy of Sciences under supervision of Professor S.B. Stechkin. Starting from 1968, Vitalii Vladimirovich works at the Sverdlovsk Division of the Steklov Mathematical Institute of the USSR Academy of Sciences (now the Krasovskii Institute of Mathematics and Mechanics, Ural Branch of Russian Academy of Sciences (IMM UB RAS)). Starting from 1970, he also teaches at Ural State University (now Ural Federal University (UrFU)), where he headed the Department of Mathematical Analysis and Function Theory (now Department of Mathematical Analysis). At present, Arestov is a professor at UrFU and a leading researcher at the Department of Approximation and Applications of IMM UB RAS.

Arestov is a prominent specialist in function theory. He has profound fundamental results on the best approximation of operators by operators of a simpler structure, in particular, on the best approximation of unbounded operators by bounded ones and on related problems on the smallest constants in inequalities between norms of derivatives of differentiable functions, on the best approximation of one class of differentiable functions by another class of smoother functions, and optimal recovery of unbounded operators on classes of elements given with error. Recently, in this topic, he solved the problem on the best uniform approximation on the numerical axis of the first-order differentiation operator on the class of functions with a bounded second derivative by bounded linear operators acting from the space of functions with a summable Fourier transform to the space of continuous functions. He also obtained the related exact Kolmogorov-type inequality between the uniform norm of the derivative of a function, the $L$-norm of the Fourier transform of the function, and the $L_{\infty}$-norm of its second derivative. In addition, Arestov and M.A. Filatova found a solution to the long standing problem of the best approximation of the differentiation operator by linear bounded operators on the class of twice differentiable functions in the space $L_{2}$ on the semi-axis. He obtained important results on the extremal properties of polynomials; in particular, an amazing result widely known to specialists in the theory of extremal problems about the best constant in the Bernstein inequality for trigonometric polynomials in the space $L_{p}$ for $0 \leq p<1$. A method has been created for studying extremal problems for polynomials in the space $L_{p}$ and in the more general case for Orlicz type $\varphi$-spaces. Fundamental results were obtained in extremal problems for positive definite functions, including problems related to the exact Jackson inequalities for the best approximations of functions by polynomials and entire functions as well as problems of Delsarte and Turan, which are related to discrete extremal geometric problems (some of these results were obtained together with Arestov's students A.G. Babenko and E.E. Berdysheva). In recent years, in a series of papers (together with M.V. Deikalova and others), a new approach, based on the properties of the generalized translation operator, has been developed to the study of extremal problems for polynomials and entire functions; subtle properties of generalized translation operators in spaces with different weights have been thoroughly investigated.

Arestov is the author of 85 scientific papers and several textbooks. The list of his main publications can be found at http://work.imkn.urfu.ru/arestov/index_en.html.

Arestov is one of the leading lecturers of UrFU in continuous mathematics. His department provides a high level of training of specialists in function theory, contributing to the development of scientific cooperation between UrFU and IMM UB RAS. His perceptive and comprehensible lectures form an interest in mathematics; the diverse and relevant topics of special courses attract students, many of whom continue to conduct research under Arestov's supervision. Eleven of his students successfully defended their dissertations, one of them has already become a Doctor of Physics and Mathematics, and another one holds Habilitation degree in Germany.

Arestov was a member of the editorial boards of leading scientific journals Mathematical Notes and East Journal on Approximations. He is currently the deputy editor-in-chief of Ural Mathematical Journal. Arestov took an active part in organizing and conducting a number of high-level 
international conferences as co-chairman or member of their organizing committees and program committees. Two of them are regular: triennial International Conference Algorithmic Analysis of Unstable Problems (1995-2014) and annual International S.B. Stechkin's School-Conference on Function Theory (from 1971 to the present).

August 1-10, 2018, the 43d International S.B. Stechkin's School-Conference on Function Theory dedicated to the 75th birthday of Professor Vitalii Vladimirovich Arestov was held in Kyshtym, Chelyabinskaya oblast. It was organized by UrFU and IMM UB RAS.

The collectives of Ural Federal University and the Institute of Mathematics and Mechanics, the editorial board of Ural Mathematical Journal, friends, colleagues, and students, heartily congratulate Vitalii Vladimirovich on his glorious jubilee and wish him further success in scientific, educational, and social activities, good health, and great personal happiness. 